\title{
IMPORTÂNCIA COMERCIAL E DE SUBSISTÊNCIA DA PESCA PARA COMUNIDADES DA VÁRZEA DO BAIXO AMAZONAS
}

\author{
Neriane Nascimento da Hora ${ }^{1}$, Álvaro dos Santos Moreira
}

1Doutoranda do Programa de Pós-Graduação em Sociedade, Natureza e Desenvolvimento (neri.dahora@gmail.com), UFOPA, Santarém, Pará, Brasil ${ }^{2}$ Graduando do Bacharelado Interdisciplinar em Ciências e Tecnologia das Águas. UFOPA, Santarém, Pará, Brasil

Recebido em: 04/10/2019 - Aprovado em: 30/11/2019 - Publicado em: 15/12/2019 DOI: 10.18677/EnciBio_2019B34

\begin{abstract}
RESUMO
Os ribeirinhos da várzea possuem uma economia diversificada, baseada na produção agrícola, pesqueira e pecuarista, sendo que a pesca é responsável pelo maior impacto na renda familiar. No entanto, pode-se distinguir entre comunidades em que a atividade de pesca é mais orientada para o comércio e comunidades em que os pescadores comercializam sua produção, porém em menor escala que o primeiro grupo. Este estudo desenvolvido em duas comunidades de várzea do Baixo Amazonas, localizadas no município de Santarém, por meio do monitoramento da pesca. Os resultados apontaram que as diferenças do grau de orientação econômica estão assentadas principalmente, em aspectos quantitativos. Verificou-se diferenças entre meios de produção, grau de seletividade das espécies exploradas, produção e esforço pesqueiro e destino da produção. Compreender as diferenças locais é importante para subsidiar o processo de gestão participativa, baseado nos acordos de pesca, o qual envolve sistema de lagos partilhado por diferentes comunidades.
\end{abstract}

PALAVRAS-CHAVE: Lagos de várzea. Pescadores ribeirinhos. Produção artesanal.

\section{COMMERCIAL AND SUBSISTENCE IMPORTANCE OF FISHERIES IN FLOODPLAIN COMMUNITIES OF LOWER AMAZON}

\begin{abstract}
Floodplain riverine people have a diversified economy, based on agriculture, cattle ranching, and fisheries, and this last one has a great impact on family income. However, we can distinguish between communities that fisheries are more oriented for commercial aspects and communities that fishers sell their production, but in a low scale than the first group. This study was developed in two floodplain communities of Lower Amazon, located in Santarém municipality and it was made by fisheries monitoring. The results show that fisheries' economic orientation scale is based mostly on quantitative aspects. It was verified differences between production means, species selectivity, fishing effort, production volume and production destination. To understand about variation in these aspects is important to support the participative management, based on fishing accords, which involves shared lakes systems by different communities.
\end{abstract}

KEYWORDS: Floodplain lakes. Riverine fishers. Artisanal production. 


\section{INTRODUÇÃO}

No contexto das comunidades ribeirinhas de várzea, mudanças no cenário econômico na segunda metade do século XX alteraram a importância da atividade pesqueira na economia familiar. Com a falência do mercado da juta, os ribeirinhos aos poucos abandonaram o cultivo de juta e passaram a investir mais tempo e recursos na pesca. Assim, esta deixa de ter um caráter somente de subsistência para tornar-se uma alternativa de renda às famílias ribeirinhas (MCGRATH et al., 1993).

O pescador artesanal, em geral, é detentor dos seus meios de produção, canoa e apetrechos, que explora locais de pesca próximos à sua residência, notadamente os lagos de várzea (MCGRATH et al., 1993; 1998), mas pescam também nos rios e canais, caso sejam próximos (CASTELLO et al., 2013). Parte de sua produção é destinada ao consumo familiar e outra parte é comercializada. A composição destas pescarias compreende uma ampla diversidade de espécies, com destaque para os peixes de escamas, que são espécies preferidas pelo consumo local, portanto abastem os mercados regionais (CERDEIRA et al., 2000; ALMEIDA et al., 2001).

Os grupos domésticos da várzea amazônica, além da pesca, desenvolvem outras atividades ao longo do ano, agricultura, criação de animais, de pequeno e grande porte e prestação de serviços temporários. A economia familiar é, portanto, baseada na combinação de diversas atividades produtivas, muito embora a pesca possua o maior impacto na composição da renda do ribeirinho em geral (SCHONS, 2018).

A despeito disso, a importância econômica da pesca é variável entre as famílias de várzea, o que depende, por exemplo, da composição familiar, histórico de uso dos recursos, entre outros. Em termos comunitários, o grau de orientação econômica da pesca é influenciado por um conjunto de fatores, desde a proximidade com o mercado urbano e o tamanho populacional (KEPPELER et al., 2018). Nesse sentido, realizou-se um estudo sobre a pesca exercida em comunidades ribeirinhas objetivando descrever o perfil da atividade pesqueira de cada comunidade e, assim, identificar semelhanças e diferenças que podem relacionar-se mais à pesca de subsistência e/ou comercial.

\section{MATERIAL E MÉTODOS}

Área de Estudo

Está situada na região do Baixo Amazonas - BAM, que compreende ao trecho do rio Solimões-Amazonas entre a fronteira dos estados do Pará e Amazonas até a foz do rio Xingu. Esta região abrange treze municípios, entre eles, o município de Santarém, localizado no oeste do estado do Pará. Santarém é o principal ponto de desembarque do Baixo Amazonas e o terceiro principal da bacia Amazônica. É composto de dez regiões pesqueiras, dentre as quais se destacam as regiões do Tapará, Lago Grande do Curuai e Maicá-Ituqui (PEREIRA et al., 2019).

Este estudo foi realizado em duas regiões de pesca, Tapará e Aritapera. A região do Tapará é composta por dez comunidades e a região do Aritapera por 13 comunidades. Especificamente, os dados foram coletados na comunidade Tapará Miri (TM) (2० 23' 22,858"'" S; $\left.54^{\circ} 31^{\prime} 40,766^{\prime \prime \prime} \mathrm{W}\right)$, situada na região pesqueira do Tapará, e na comunidade Água Preta (AP) ( $2^{\circ} 8^{\prime} 5,303^{\prime \prime \prime}$ S; 54 $34^{\circ} 33,337^{\prime \prime \prime}$ W), situada na região pesqueira do Aritapera. 
Segundo a classificação climática de Köppen, a região apresenta clima do tipo Am (equatorial úmido) e a precipitação anual nos anos de 2015 e $2017^{1}$ foram iguais a 2030,9 mm e 2090,7 mm (Estação Santarém 254000), respectivamente (ANA, 2019). Ambas as comunidades compõem a região de várzea do município Santarém, que corresponde às planícies alagáveis nas laterais do rio Amazonas. É uma área que apresenta terrenos baixos, cuja altitude é de aproximadamente $20 \mathrm{~m}$ acima do nível do mar.

\section{Coleta de Dados}

Foram realizadas entrevistas recordatórias sobre a atividade de pesca, com no máximo dois após a pescaria, durante os últimos sete dias consecutivos de cada mês durante um ano, de outubro/2016 a setembro/2017². Selecionou-se 14 famílias em cada comunidade, de acordo com a disponibilidade e aceitação das mesmas pelo estudo, isto é, após livre consentimento e esclarecido. Os dados coletados sobre as pescarias eram anotados em formulário de registro diário da atividade de pesca, consumo e venda. Esta metodologia foi adotada por diversos estudos no Baixo Amazonas (MCGRATH et al., 1998; CASTELLO et al., 2011; 2013).

Em conjunto com métodos quantitativos, realizou-se o levantamento de informações qualitativas por meio de observação direta, com registros em caderno de campo, a fim de compreender o contexto dos dados de monitoramento da pesca (BARCLAY et al., 2017). Neste sentido, realizaram-se excursões a campo sempre que possível, nos diversos períodos do ano.

\section{Análise de Dados}

Utilizou-se o pacote de estatística descritiva para calcular medidas de tendência centrais, desvio padrão, valores mínimos e máximos, frequência absoluta e percentual em relação às variáveis analisadas.

Como parâmetro de produtividade pesqueira, utilizou-se a captura por unidade de esforço (CPUE), sendo calculada para cada pescaria realizada. Para o cálculo de CPUE, dividiu-se a produção $(\mathrm{kg})$ pelo esforço pesqueiro, sendo que o esforço pesqueiro é calculado multiplicando o número de pescadores envolvidos na pescaria pelo tempo dispendido com a atividade, incluindo-se o tempo de viagem até o local de pesca (MCCLUSKEY; LEWISON, 2008).

Utilizou-se como unidade do tempo de pescaria tanto horas, como dias, obtendo-se, dessa forma, a CPUE (dia), isto é, baseada no número de dias gastos na pescaria, e a CPUE (hora) que considera a quantidade de horas dispendidas na pescaria (MCCLUSKEY; LEWISON, 2008). Também se efetuou o cálculo da Captura por Viagem que diz respeito a soma dos quilos produzidos em cada pescaria.

Estimou-se a produção mensal de pescado em cada comunidade. Para tal, multiplicou-se a captura por pescador por dia (CPUE) (média e mediana) pelo número de pescadores da comunidade e multiplicou-se pela frequência mensal de pesca. A frequência mensal de pesca foi obtida a partir da extrapolação da frequência semanal de pesca, baseado em Cerdeira et al., (2000).

\footnotetext{
${ }^{1}$ Para o ano de 2016, os dados sobre precipitação pluviométrica da Agência Nacional de Águas ANA estão incompletos.

${ }^{2} \mathrm{Na}$ comunidade Água Preta não foi possível realizar entrevistas durante o mês de janeiro de 2017 devido um imprevisto com um dos entrevistadores, somado a impossibilidade de substituição do entrevistador em tempo hábil para a coleta das informações.
} 


\section{RESULTADOS}

Várzea: ambiente, ecologia humana e organização social

A várzea corresponde às planícies laterais dos rios de águas brancas que são periodicamente inundadas devido à elevação do nível do rio durante parte do ano (JUNK et al., 1989). O pulso de inundação é regido pela diferença na distribuição das chuvas ao longo do ano, o que resulta no aumento e diminuição do nível dos rios, isto é, um ciclo anual de cheia e seca.

O nível do rio, próximo à Santarém, atinge seu máximo entre os meses de maio e junho e diminui a partir de julho, atingindo seu nível mais baixo entre os meses de outubro/novembro; e a partir de dezembro começa a subir novamente. $O$ ciclo de chuvas é aproximadamente igual ao regime de inundação, podendo ser divididos em um período chuvoso (inverno amazônico), que dura mais ou menos cinco meses, e um período seco (verão amazônico), de mais ou menos sete meses (MCGRATH et al., 1993).

Este padrão cíclico anual do nível do rio e de chuvas modifica a paisagem ao longo do ano. Os principais elementos desta paisagem, rio, lago, restinga, campo, canais e floresta, ora estão isolados, ora formam um ambiente amplo e integrado (JUNK et al., 1989; CASTRO, 2016).

Os lagos são a principal unidade de paisagem do ambiente varzeiro, tendo grande importância para pesca de subsistência e comercial das famílias ribeirinhas. Os lagos, em geral, estão localizados no interior da comunidade, logo atrás dos terrenos de moradia dos ribeirinhos. Em frente aos terrenos, localizam-se os rios e/ou canais, os quais também são utilizados como áreas de pesca das famílias ribeirinhas (MCGRATH et al., 1993; CASTRO, 2016).

As restingas são terrenos altos margeados pelos rios, canais e/ou lagos, na qual se constroem as moradias e desenvolve-se o plantio de roças e outros cultivos de ciclo curto. Os campos naturais situam-se entre as restingas e os lagos e são utilizados como pastagem pelos criadores de animais, principalmente gado, mas também para a agricultura. Nos terrenos mais altos, adjacentes aos lagos, está a floresta de várzea (MCGRATH et al., 1993; CASTRO, 2016).

As comunidades são constituídas de dezenas a centenas de famílias e são representadas por uma associação comunitária. Ainda, alguns pescadores são associados às Colônias de Pesca, representados por um núcleo de base (CASTRO, 2016). Na comunidade AP residem 65 famílias, sendo que 95 moradores são associados à Associação Comunitária da Água Preta (ACAP) e o núcleo de base é composto por 55 pescadores (as). Na comunidade TM vivem 89 famílias, sendo que 110 residentes são sócios da Associação de Moradores de Tapará Miri (AMOTAM) e existem 84 sócios do núcleo de base da comunidade.

\section{Características da Pesca}

Esta análise compreende o período de outubro/2016 a setembro/2017, no qual se obteve 2254 entrevistas. As entrevistas diárias nem sempre resultaram em registros de pescaria ${ }^{3}$, portanto, do total de entrevistas com registros de atividade pesqueira foram 1635, sendo 599 pescarias na comunidade AP e 1036 pescarias na comunidade TM.

\footnotetext{
3 O formulário utilizado nas entrevistas do monitoramento pesqueiro compreendiam outras informações além do registro da atividade de pesca, que são: consumo familiar e registros de venda de pescado.
} 


\section{Embarcação}

As embarcações utilizadas na pesca pelas comunidades de várzea no Baixo Amazonas são: bote, canoa, rabeta e bajara - todas de madeira. Os botes, também denominados cascos, são as menores embarcações, em geral, não acopladas a motores, sendo preciso utilizar remos para a navegação. Canoa é o termo mais comum para embarcação utilizada pelos ribeirinhos na região amazônica e elas são um pouco maiores que os botes.

As rabetas, especificamente, dizem respeito ao motor colocado nas canoas, mas em geral, quando os pescadores referem-se que utilizaram rabeta, isto significa que se trata de uma canoa motorizada, isto é, sem necessidade de remos. A bajara é uma canoa maior, em média $7 \mathrm{~m}$ (ISAAC et al., 2016) e podem ter cobertura solar.

Os pescadores das duas comunidades utilizam com maior frequência os botes, sobretudo na comunidade TM $(77,1 \%)$. As canoas apresentaram percentuais de uso aproximados nas duas comunidades, em torno de $10 \%$. As rabetas ${ }^{4}$ são mais utilizadas na comunidade AP (28,4\%) em relação à comunidade TM (10\%). As bajaras são pouco utilizadas pelos pescadores entrevistados, sobretudo na comunidade TM em que somente $1,3 \%$ das pescarias foram realizadas em bajaras. $\mathrm{Na}$ comunidade AP, entretanto, as bajaras representaram frequência de uso mais elevada $(13,8 \%)$.

\section{Arreios (apetrechos)}

Foram identificados sete diferentes arreios de pesca nas pescarias das comunidades Água Preta e Tapará Miri, são eles: 1) malhadeira, 2) tarrafa, 3) caniço, 4) linha de mão, 5) flecha, 6) arpão e 7) espinhel, baseado na classificação de Cruz et al. (2017).

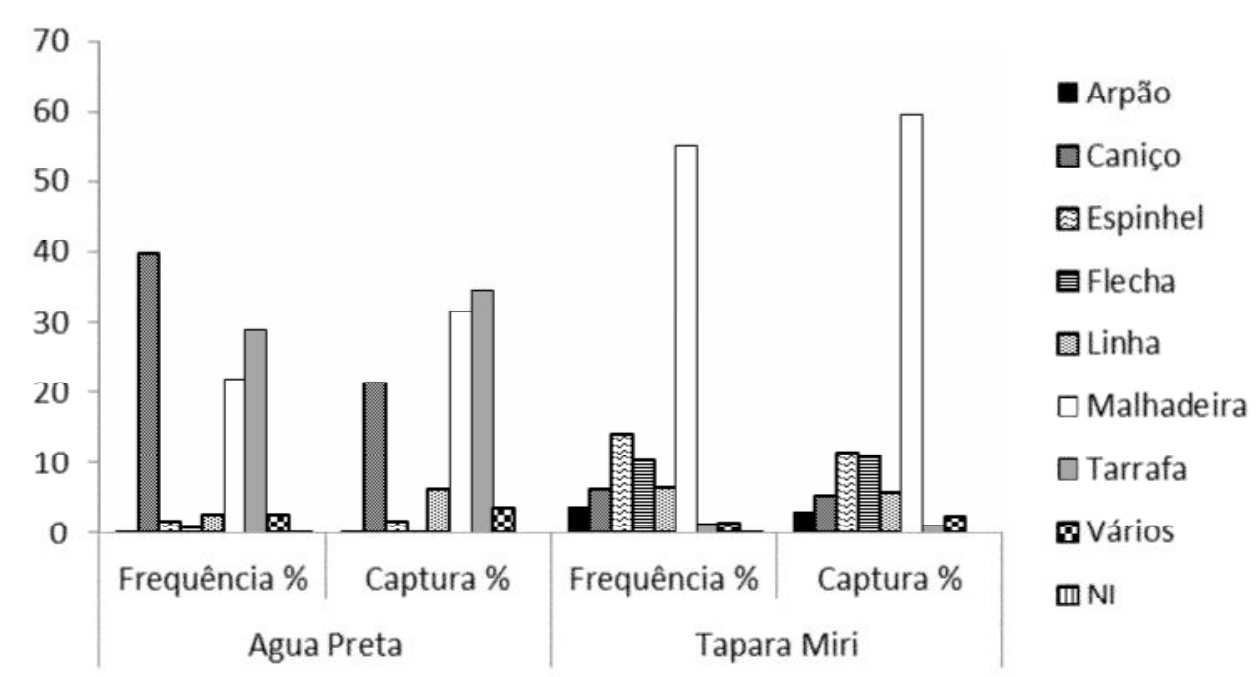

FIGURA 1 - Frequência percentual do uso de arreios e captura percentual por arreio nas comunidades Água Preta e Tapará Miri. Fonte: Entrevistas recordatórias.

$\mathrm{Na}$ comunidade AP, a arte de pesca mais utilizada pelos pescadores foi 0 caniço $(39,9 \%)$, em seguida a tarrafa e a malhadeira. No entanto, o maior volume de

\footnotetext{
${ }^{4}$ botes adaptados para acoplação de motores, o que ainda é pouco comum, foram classificados na categoria rabetas.
} 
captura foi conseguido com a tarrafa $(34,7 \%)$ e depois a malhadeira $(31,7 \%)$. $\mathrm{Na}$ comunidade TM, a malhadeira foi o principal arreio utilizado nas pescarias $(55,5 \%)$, obtendo também o maior volume percentual de captura $(59,7 \%)$, seguida do espinhel e a flecha que representaram a segunda e terceira maior porcentagem tanto de uso quanto de volume capturado pelos pescadores. As artes de fisgar (arpão e flecha) são mais utilizadas na comunidade TM (14\%) em relação à comunidade AP (1,2\%) e representam uma captura de $13 \%$ no TM. A combinação de mais um arreio em uma pescaria foi pouco frequente em ambas as comunidades (Figura 01).

\section{Ambientes de Pesca}

Os ambientes foram caracterizados com base na literatura e na denominação local, listados a seguir: baixa, igarapé, igapó, canal, lago, poço, restinga e rio. As baixas são áreas inundadas quase a maior parte do ano. Os igarapés são cursos d'água que conectam trechos do rio principal. Os igapós são as áreas de floresta inundada. Os canais conectam os lagos aos demais tipos de cursos d'água (CASTELLO, 2008). Os poços são os locais mais fundos dos lagos que raramente secam. As restingas são os terrenos mais elevados, mas também suscetíveis de inundação durante o período de águas elevadas (MCGRATH et al., 1993; CASTELLO, 2008). Por fim, o rio diz respeito ao curso d'água principal, a calha maior que se mantém durante o ano inteiro.

O lago foi o ambiente mais frequentado pelos pescadores das duas comunidades, representando mais de $50 \%$ das pescarias em ambas as comunidades. Na comunidade TM, a restinga foi o segundo $(22,3 \%)$ ambiente mais utilizado pelos pescadores; por outro lado, o segundo ambiente em importância de uso na comunidade AP foi o igapó $(33,2 \%)$. Os demais ambientes, com exceção dos igarapés $(16,4 \%)$ na comunidade TM, representaram menos de $10 \%$ nas pescarias de ambas as comunidades.

Os lagos também concentraram mais da metade do volume capturado em ambas as comunidades. Na comunidade TM, a captura nos ambientes fluviais (rios, igarapés e canais) correspondeu a $23,8 \%$ da produção contra $13,1 \%$ na comunidade AP. Nos ambientes alagados, o volume de produção nas comunidades TM e AP foram próximos, iguais a $24 \%$ e $21 \%$, respectivamente.

\section{Esforço pesqueiro}

Nas comunidades estudadas, as pescarias ocorrem com até dois pescadores por embarcação. Na comunidade AP, no entanto, a maioria das viagens de pesca $(97,8 \%)$ é realizada de forma solitária. Na comunidade TM, as viagens de pesca com um pescador ou dois pescadores ocorrem aproximadamente na mesma proporção, sendo $49,5 \%$ das viagens em regime de parceria. O tempo médio de pesca (inclui o tempo de viagem) foi igual a 8,2 $\pm 3,3 \mathrm{~h}$ e $5,4 \mathrm{~h} \pm 2,9 \mathrm{~h}$ nas comunidades TM e AP, respectivamente.

Na comunidade TM, a frequência semanal média de pesca foi de 0,88 , igual a 6,2 dias por semana, totalizando 321 dias de pesca no ano. Por outro lado, na comunidade AP obteve-se uma frequência semanal média igual 0,56 , equivalendo a 3,9 dias de pesca por semana e 202 dias de pesca ao ano.

Produção pesqueira

Nas comunidades AP e TM, obtiveram-se $3.962,2 \mathrm{~kg}$ (11 meses) e $25.895,4$ $\mathrm{kg}$ (12 meses) de produção de pescado monitorado, respectivamente, com destaque 
para o segundo semestre do ano que foi mais produtivo em ambas as comunidades, apesar disso a TM também teve uma produção relevante em fevereiro.

A captura por viagem de pesca é bastante variável, sendo que a produção foi nula em algumas viagens, mas houve pescarias de até $275 \mathrm{~kg}$ capturados. As viagens que resultaram em zero quilos capturados foram iguais a $1,25 \%$ (13 pescarias) na comunidade TM e $0,17 \%$ (01 pescaria) na comunidade AP. Por outro lado, capturas por viagem com mínimo de $20 \mathrm{~kg}$ correspondeu a $55 \%$ do total da comunidade TM e 5,0\% na comunidade AP (Tabela 01).

Como os valores de captura por viagem e captura por unidade de esforço apresentaram uma distribuição assimétrica $(\mathrm{W}=0,6905 ; \mathrm{p}=1,318 \mathrm{E}-47 / \mathrm{W}=0,6469$; $p($ normal $)=9,875 E-50)$, calculou-se os valores medianos de captura, mas também apresentam-se os valores médios para efeitos comparativos. A captura por viagem e a captura por unidade de esforço foram maiores (mediana e média) na comunidade $\mathrm{TM}$ em relação à comunidade AP (Tabela 01).

TABELA 1- Tabela descritiva dos valores medianos, médios, desvio padrão (DP), mínimo e máximo das capturas por viagem de pesca e das capturas por unidade de esforço (CPUE).

\begin{tabular}{lcccccc}
\hline & \multicolumn{2}{c}{ Captura por viagem } & \multicolumn{2}{c}{ CPUE (dias) } & \multicolumn{2}{c}{ CPUE (horas) } \\
\hline & Água Preta & Tapará Miri & Água Preta & Tapará Miri & Água Preta & Tapará Miri \\
\hline Mediana & 4,0 & 20,0 & 4,0 & 14,0 & 0,9 & 1,8 \\
Média & 6,6 & 25,0 & 6,6 & 18,7 & 1,6 & 2,6 \\
D.P & 7,8 & 22,6 & 7,8 & 18,9 & 2,1 & 2,9 \\
Mínimo & 0,0 & 0,0 & 0,0 & 0,0 & 0,0 & 0,0 \\
Máximo & 75 & 245,0 & 75 & 245 & 17 & 40,8 \\
\hline
\end{tabular}

Fonte: Entrevistas recordatórias

A partir da captura mediana por pescador por dia e a frequência anual de pesca (citada no tópico anterior), cada pescador produz 4,5 t e 0,8 t de peixes anualmente nas comunidades TM e AP, respectivamente. Considerando o total de pescadores por comunidade (citado no tópico 3.1), obtém-se uma produção anual total igual a 377 t e 44 t nas comunidades TM e AP, respectivamente. Ao considerarse a captura média (para efeitos comparativos com outros estudos), cada pescador produz cerca de 6,0 t e 1,3 t ao ano nas comunidades TM e AP, respectivamente. Com base também na captura média, estima-se que a produção total anual das comunidades TM e AP, respectivamente, são iguais a 504 t e 73 t, aproximadamente.

Grupo de espécies exploradas

29 categorias de espécie ${ }^{5}$ foram identificadas em ambas as comunidades, sendo que dessas, 28 espécies foram registradas nas pescarias da comunidade Água Preta e 19 na comunidade Tapará Miri. No entanto, em ambas as comunidades oito espécies concentraram $80 \%$ da produção total. Dentre as oito espécies, seis foram comuns a ambas as comunidades (tambaqui, surubim, pirapitinga, pacu, aruanã e acari), mas diferiram quanto à posição relativa de contribuição ao volume total (Figuras 2 e 3).

\footnotetext{
${ }^{5}$ Espécie, neste estudo, não se refere à classificação biológica, mas a denominação regional/popular dada aos peixes capturados. Neste sentido, em alguns casos, um nome popular pode representar várias espécies na classificação científica, bem como uma única espécie na classificação científica pode receber vários nomes populares.
} 


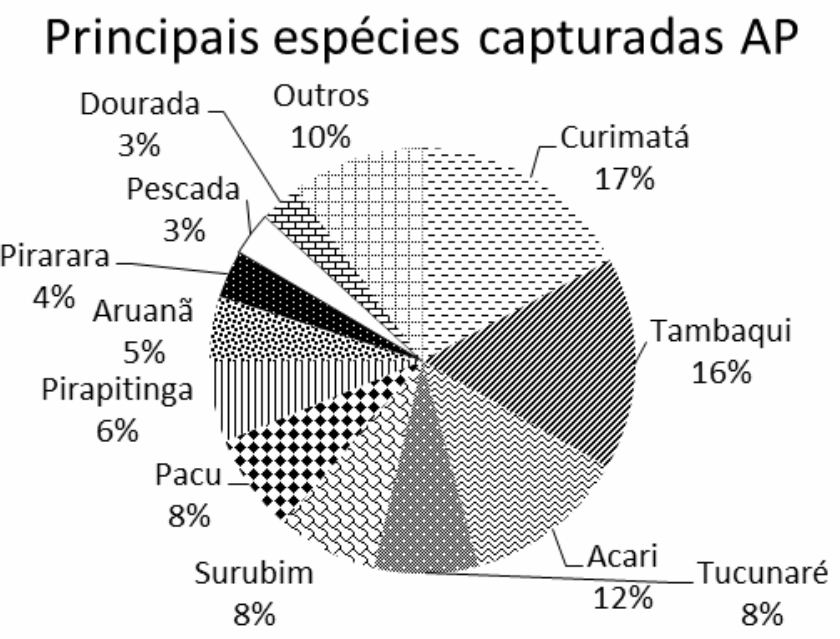

FIGURA 2 - Captura $(\mathrm{kg})$ percentual das principais etnoespécies registradas nas pescarias da comunidade AP.

Fonte: Entrevistas recordatórias.

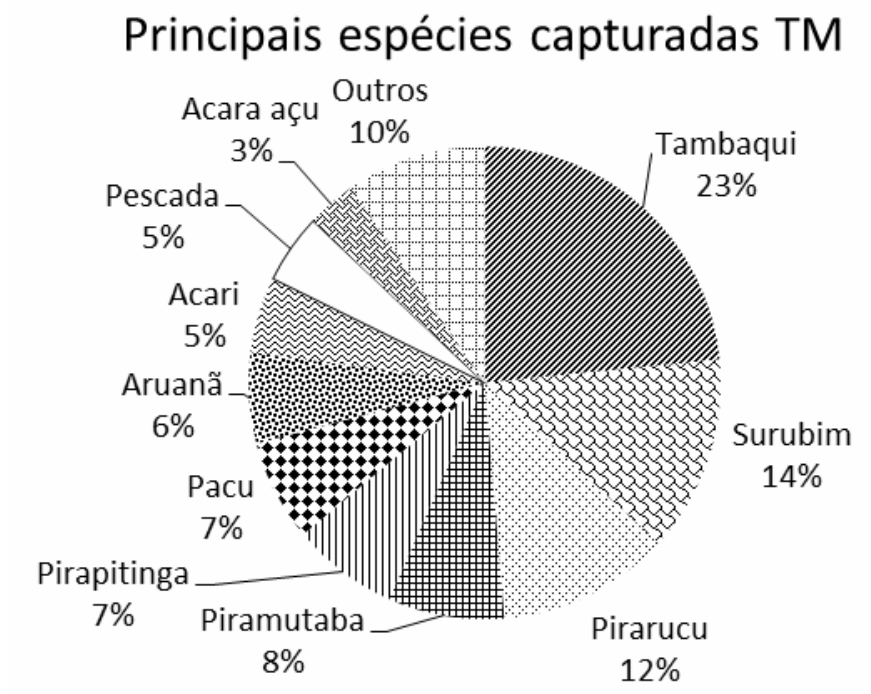

FIGURA 3- Captura (kg) percentual das principais etnoespécies registradas nas pescarias da comunidade TM.

Fonte: Entrevistas recordatórias.

Destino da produção

$\mathrm{Na}$ comunidade AP, a maioria das viagens de pesca $(63,8 \%)$ é realizada unicamente para a subsistência familiar. Por outro lado, na comunidade TM, a maioria das pescarias $(48,1 \%)$ é realizada tanto para suprir o consumo familiar como para a venda de peixe, em segundo lugar, as pescarias unicamente comerciais foram mais importantes, representando $42,5 \%$ das viagens de pesca.

$\mathrm{Em}$ relação à quantidade produzida destinada para o consumo e para a venda, tomando como base somente as pescarias voltadas unicamente para a venda e/ou para o consumo, verificou-se que na comunidade AP, a quantidade consumida foi maior, 1,4t, do que a quantidade vendida, $0,8 \mathrm{t}$. Na comunidade TM, por outro lado, a quantidade vendida foi igual a 9,6 t e a consumida foi igual a 1,0 t, isto é, a quantidade vendida é nove vezes maior que a quantidade consumida (Tabela 02). 
TABELA 2 - Produção $(\mathrm{kg})$ em relação ao destino das pescarias nas comunidades Água Preta e Tapára Miri entre out/16 a set/17.

\begin{tabular}{lccc}
\hline & Água Preta & Tapará Miri & Total \\
\hline Consumo & 1429,5 & 1018,0 & 2447,5 \\
Consumo e Venda & 1710,4 & 15199,4 & 16909,8 \\
Venda & 821,8 & 9604,0 & 10425,8 \\
Não identificado & 11,0 & 74,0 & 85,0 \\
Total & 3972,6 & 25895,4 & 29868,0 \\
\hline
\end{tabular}

Fonte: Entrevistas recordatórias.

\section{DISCUSSÃO}

A pesca é a atividade prevalente entre aquelas exercidas pelas famílias das comunidades estudadas, mas há diferenças de ordem de importância econômica da atividade pesqueira entre as comunidades. Pode-se dizer que a pesca tem maior importância econômica para as famílias da comunidade Tapará Miri em relação à comunidade Água Preta.

Apesar dessas diferenças, a atividade pesqueira é essencialmente artesanal em ambas as comunidades, isto é, as características que definem pesca artesanal são mantidas independente do grau de importância econômica. Entretanto, é possível apontar os aspectos no que diz respeito aos meios de produção empregados, arreios, ambientes explorados, esforço pesqueiro, produção pesqueira e grupo de espécies exploradas, sejam qualitativas ou quantitativas, em algum grau.

\section{Embarcação}

$\mathrm{Na}$ AP e TM todos os pescadores entrevistados pescam em suas pequenas embarcações, preferencialmente a remo. $O$ uso de pequenas embarcações a remo também é encontrado em estudos mais antigos e atuais sobre a pesca na Amazônia (GOULDING, 1979; SMITH, 1979; HALLWASS et al., 2011; CASTELLO et al., 2013). Portanto, apesar de incentivos à modernização do setor pesqueiro na Amazônia em década recentes, incluindo a pesca de pequena escala (MCGRATH et al., 1993; MCGRATH et al., 2015), a pesca artesanal apresentou poucas inovações no que diz respeito aos equipamentos e estratégias empregadas (MCGRATH et al., 1998).

A manutenção do uso de botes e canoas na pesca pode estar associada à maior acessibilidade a ambientes, como exemplo, lagos mais rasos, com bastante vegetação (aningais), floresta alagada com bastante troncos, entre outros. Pode ser associado também às restrições sobre o uso de motores nos locais de pesca, sobretudo lagos e floresta alagada, pois os motores são considerados como "afugentadores" dos peixes.

\section{Arreios}

O uso de artes simples e variadas, utilizadas ou não de forma conjunta, é uma característica da pesca artesanal na Amazônia (CERDEIRA et al., 2000; LOPES et al., 2016). Apesar disso, as redes de emalhe têm grande importância na pescaria nas duas comunidades, seja como arreio mais utilizado, seja como arreio que mais contribuiu para a produção pesqueira. Na comunidade AP, por exemplo, embora o caniço tenha sido o principal arreio empregado, a malhadeira representou a maior proporção de captura. As malhas de fibra sintética foram introduzidas em décadas recentes e têm predominado nas pescarias amazônicas (CASTELLO et al., 2013; LOPES et al., 2016; SILVANO et al., 2016).

Mas há diferenças quanto à intensidade do uso de malhadeira entre as comunidades dependendo do grau de importância econômica da pesca. A 
malhadeira foi o principal arreio na comunidade TM, com maior orientação comercial da pesca, enquanto que na comunidade AP, na qual os pescadores têm orientação mais voltada para subsistência, a malhadeira teve menor relevância (MCGRATH et al., 1998; CASTELLO et al., 2013).

\section{Ambientes de pesca}

Os ambientes lacustres são bastante importantes para a pesca artesanal na Amazônia (BRELAZ et al, 2018; PEREIRA et al., 2019), como evidenciado neste estudo. Os lagos não somente concentraram maior número de viagens, como também o maior volume de produção nas comunidades estudadas. Em geral, esse padrão é diferente no mercado de Santarém, devido ao aporte da produção dos barcos comerciais que exploram a calha dos rios. Nos mercados, a despeito da maioria dos desembarques pesqueiros serem provenientes de pescarias em lagos, a maior produção é proveniente da pesca nos rios. Os barcos comerciais devido ao maior poder de captura e de explorar áreas mais distantes acabam tendo bastante impacto na produção total (ALMEIDA et al., 2001; ALMEIDA et al., 2014).

\section{Esforço pesqueiro}

O número de pescadores máximo em cada pescaria foi igual a dois em cada comunidade. No entanto, a pesca de parceria foi mais importante na comunidade TM do que na comunidade AP. O regime de parceira é comum entre membros da mesma família, pois ao se utilizar mão de obra familiar, busca-se garantir maior produção sem a necessidade de divisão de lucros. A comunidade AP, pelo maior caráter de subsistência da pesca, em geral, não investe um número grande de pessoas na atividade, pois isso significaria reduzir mão de obra em outras atividades.

Com relação ao tempo dispendido com a pesca, ele está dentro do esperado para a pesca artesanal nos trópicos, isto é, entre sete e oito horas (MCGRATH et al., 1998; CERDEIRA et al., 2000). Embora a pesca artesanal, via de regra, ocorra dentro de 24 horas, pode-se notar uma diferença entre pescadores/comunidades comerciais e de subsistência. Na comunidade AP predomina a pesca de subsistência, assim os pescadores costumam realizar viagens mais curtas, pois à medida que conseguem capturar para suprir a demanda de alimento retornam para sua residência. Os pescadores do $\mathrm{TM}$, por outro lado, gastam mais tempo na pescaria na tentativa de aumentar o volume de captura e garantir maior retorno econômico (MCGRATH et al., 1998).

A frequência mensal da pesca também indica o grau de importância econômica da pesca para as famílias (MCGRATH et al., 1998). Nisto pode-se reforçar a maior importância econômica da pesca na comunidade TM, em que no mínimo $67,7 \%$ do mês (de 30 dias) foram investidos na pesca, sem contar os dias que são realizadas atividades de reparo e conserto de arreios. Por outro lado, na comunidade AP, em média investem 16,9 dias por mês na atividade pesqueira, indicando menor grau de importância econômica da pesca nesta comunidade.

Produção pesqueira

Nas comunidades, o período de águas baixas (vazante e seca) foi mais favorável à produção pesqueira, padrão comum da pesca nos rios de águas brancas (LOPES et al., 2016). Sobre isso, os varzeiros costumam classificar o "verão amazônico", segundo semestre do ano, que começa mais ou menos com a diminuição do nível do rio como período de abundância, isto é, de safra. O "inverno 
amazônico", por outro lado, é considerado um período de escassez (MCGRATH et al., 1993).

A captura por viagem de pesca também revelou que os pescadores da comunidade AP apresentaram maior caráter de subsistência em relação aos pescadores da comunidade TM. Em geral, pescadores de subsistência capturam poucos quilos por viagem (até $05 \mathrm{~kg}$ ), mas poucas capturas iguais a zero. Por outro lado, pescadores mais comerciais apresentam maior número de capturas iguais a zero, mas ao mesmo tempo muitas viagens com capturas acima de $20 \mathrm{~kg}$ (MCGRATH et al., 1998).

Quanto ao rendimento das pescarias, Castello et al. (2013) ao avaliar pescarias de oito comunidades do Baixo Amazonas entre 1995 e 1997 e 2003 e 2007, calculou CPUE média entre as comunidades comerciais igual a $1,38 \mathrm{~kg}$ pescador $^{-1}$ hora $^{-1}$, portanto um valor menor quando comparado a CPUE média da comunidade TM $\left(2,65 \mathrm{~kg}\right.$ pescador $^{-1}$ hora $\left.^{-1}\right)$. Em relação às comunidades de subsistência, a CPUE variou entre 1,00 e 1,73 $\mathrm{kg}_{\text {pescador }}{ }^{-1}$ hora $^{-1}$, portanto, a CPUE verificada na comunidade AP $\left(1,64 \mathrm{~kg}\right.$ pescador ${ }^{-1}$ hora $\left.^{-1}\right)$ apresentou variação esperada entre as comunidades de subsistência. Pode-se dizer que a pesca artesanal quando orientada mais ao consumo familiar possui uma baixa escala de produção, por outro lado quando possui maior orientação comercial, a escala de produção tende a ser maior.

\section{Grupo de espécies exploradas}

Os desembarques pesqueiros na Amazônia são caracterizados por uma alta diversidade de espécies capturadas (HALLWASS; SILVANO, 2016). Neste estudo, somente em duas comunidades, obteve-se 29 etnoespécies registradas, sendo que algumas categorias representam mais de uma espécie na classificação científica, portanto, a quantidade real de espécies pode ser ainda maior.

Ao comparar-se o total de espécies registradas em cada comunidade, 0 Tapará Miri apresentou uma menor diversidade de espécies (19) em relação a comunidade Água Preta (28). Apesar da multi-especificidade da pesca artesanal, pode-se dizer que dependendo da orientação econômica, as pescarias podem tornar-se mais seletivas, em especial sobre espécies de maior valor comercial, como é o caso da comunidade Tapará Miri.

As três principais espécies das comunidades são Characiformes, exceto o surubim em uma das comunidades. Tal fato era esperado visto que a pesca artesanal explora sobretudo os peixes de escama e são orientadas para o mercado local (ALMEIDA et al., 2001). A composição do desembarque também reflete a predominância dos grupos de pescado na Amazônia. Characiformes (43\%) e Siluriformes (39\%) são principais grupos de espécies encontrados na bacia amazônica; os demais 18\% estão distribuídos em outros grupos de espécies (BARLETTA et al., 2016).

Destino da produção

O pescado capturado é destinado tanto ao comércio, como ao consumo familiar de ambas as comunidades. A produção destinada, em parte, para a subsistência familiar é uma das principais características da pesca artesanal (CERDEIRA et al., 2000), embora algumas comunidades tenham maior caráter comercial (CASTELLO et al., 2013). Entre as razões da manutenção da pesca de subsistência nas comunidades amazônicas pode ser atrelado ao fato que o peixe, 
em conjunto com a farinha, constitui o principal cardápio alimentar do ribeirinho (ISAAC et al., 2015).

Durante a seca, em geral, há proibição da pesca comercial nos lagos pelos acordos de pesca, mas permite-se a pesca de subsistência, a fim de garantir o sustento das famílias por meio de acordos de pesca (FERREIRA; SILVA et al., 2017).

\section{CONCLUSÕES}

A pesca realizada nas comunidades estudadas é típica das pescarias artesanais da Amazônia, multi-específica e multi-aparelho. Apesar disso, há diferenças no perfil da atividade pesqueira entre as comunidades estudadas que podem ser atreladas ao grau de orientação econômica da pesca.

Os aspectos distintivos entre a pesca comercial e de subsistência foram os seguintes: esforço pesqueiro, maior na comunidade mais comercial; seletividade das espécies, maior também na comunidade mais comercial; maior intensidade de uso de malhadeira na comunidade mais comercial; produção pesqueira e rendimento das pescarias em maiores escalas na comunidade mais comercial e, consequentemente, maior volume de produção destinado à comercialização na comunidade mais comercial. Não houve diferenças quanto à tipologia e intensidade de uso das embarcações, bem como os ambientes explorados refletem mais a disponibilidade de habitats do que alguma diferença entre o grau produção voltada ao mercado entre as comunidades.

Portanto, as dissimilaridades entre as comunidades que exercem a pesca comercial ou de subsistência dizem respeito mais a aspectos quantitativos do que qualitativos.

Este trabalho trouxe elementos que reforçam a discussão sobre a importância da pesca para as comunidades de várzea. Precisamente, ele aponta que as heterogeneidades da atividade na Amazônia se apresentam não somente ao nível macro-regional, mas também intercomunitários, e que essas diferenças, em algum ponto, estão relacionadas ao nível de importância econômica da atividade para as famílias.

\section{REFERÊNCIAS}

ALMEIDA, O. T.; MCGRATH, D. G.; RUFFINO, M. The commercial fisheries of the lower Amazon: an economic analysis. Fisheries Management and Ecology, v. 8, n. 253-269, 2001. Disponível em: <https://doi.org/10.1046/j.1365-2400.2001.00234.x>.

AGÊNCIA NACIONAL DE ÁGUAS - ANA. Sistema de Informações Hidrológicas. Disponível

em:

<http://www.snirh.gov.br/hidroweb/publico/medicoes_historicas_abas.jsf >. Acesso em: 15 de outubro de 2019.

ALMEIDA, O. T.; MCGRATH, D. G.; RIVERO, S.; FILGUEIRAS, G.; THOMAS, S. et al. Alternativa de comercialização para o pescador artisanal do Baixo Amazonas. Papers do NAEA, n. 339, p. 3-16, 2014. Disponível em< http://www.naea.ufpa.br/naea/novosite/paper/413>

BARLETTA, M.; CUSSAC, V. E.; AGOSTINHO, A. A.; BAIGÚN, C.; OKADA, E. K. Fisheries ecology in South America river basins. In: CRAIG, J. F. (Ed). Freshwater fisheries ecology. John Wiley \& Sons, 2016. 
BRELAZ, R. L.; FARIA JR., C. H.; RIBEIRO, F. R. V. Caracterização da atividade pesqueira na comunidade Vila Flexal do município de Óbidos, Pará, Brasil: subsídios para gestão dos recursos pesqueiros. Scientia Amazonia, v. 7, n. 1, p. 134-155, 2018.

BARCLAY, K.; VOYER, M.; MAZUR, N.; PAYNE, A. M.; MAULI, S. et al. The importance of qualitative social research for effective fisheries management. Fisheries Research, n. 186, p. 426-438, 2017. Disponível em: < https://doi.org/10.1016/j.fishres.2016.08.007>.

CASTELLO, L. Lateral migration of Arapaima gigas in floodplains of the Amazon. Ecology of Freshwater Fish, 17, 38-46, 2008. Disponível em:

<https://doi.org/10.1111/j.1600-0633.2007.00255.x>.

CASTELLO, L.; MCGRATH, D. G.; BECK, P. S. A. Resource sustainability in smallscale fisheries in the Lower Amazon floodplain. Fisheries Research, v. 110, p. 356364, 2011. Disponível em: <https://doi.org/10.1016/j.fishres.2011.05.002>.

CASTELLO, L.; MCGRATH, D. G.; ARANTES, C. C.; ALMEIDA, O. Accounting for heterogeneity in small-scale fisheries management: the Amazon case. Marine Policy, v. 38, p. 557-565, 2013. Disponível em: $<$ https://doi.org/10.1016/j.marpol.2012.09.001>.

CASTRO, F. Local politics of floodplain tenure in the Amazon. International Journal of the Commons, v. 10, n. 1, p. 1-20, 2016. doi: 10.18352/ijc.472

CERDEIRA, R. G. P.; RUFFINO, M. L.; ISAAC, V. J. Fish catches among riverside communities around Lago Grande de Monte Alegre, Lower Amazon, Brazil. Fisheries Management and Ecology, p. 355-374, n. 7, 2000. Disponível em: $<$ https://doi.org/10.1046/j.1365-2400.2000.007004355.x>.

CRUZ, R. E. A.; ISAAC, V. J.; PAES, E. T. A pesca da dourada Brachyplatystoma rousseauxii (CASTELNAU, 1855) na região do Baixo Amazonas, Brasil. Boletim do Instituto de Pesca, São Paulo, v. 43, n. 4, p. 474-486, 2017. Disponível em:< https://www.pesca.sp.gov.br/01_A-PESCA-DA-DOURADA-Brachyplatystomarousseauxii-_CASTELNAU-1855_-NA-REGIAO-DO-BAIXO-AMAZONASBRASIL.pdf>.

FERREIRA, R. R.; SILVA, R. E. Acordos de pesca como gestão dos recursos: o caso da Ilha São Miguel, Santarém, Pará. Amazônica: Revista de Antropologia, v. 9, n. $1, \quad$ p. 156-178, 2017. Disponível em: <https://periodicos.ufpa.br/index.php/amazonica/article/view/5487>.

GOULDING, M. Ecologia da pesca no rio Madeira. Manaus: INPA, 1979.

HALLWASS, G.; LOPES, P. F.; JURAS, A. A.; SILVANO, R. A. M. Fishing effort and catch composition of urban market and rural villages in Brazilian Amazon. 
Environmental Management, v. 47, p. 188-200, 2011. doi: 10.1007/s00267-0109584-1

HALLWASS, G.; SILVANO, R. A. M. Patterns of selectiveness in the Amazonian freshwater fisheries: implications for management. Journal of Environmental Planning and Management, v. 59, n. 9, p. 1537-1559, 2016. Disponível em: < http://dx.doi.org/10.1080/09640568.2015.1081587>.

ISAAC, V. J.; ALMEIDA, M. C.; GIARRIZZO, T.; DEUS, C. P; VALE, R. et al. Food consumption as an indicator of the conservation of natural resources in riverine communities of the Brazilian Amazon. Anais da Academia Brasileira de Ciências, v. 87, n. 4, p. 2229-2242, 2015. Disponível em: <http://dx.doi.org/10.1590/00013765201520140250>.

ISAAC, V. J.; ALMEIDA, M. C.; CRUZ, R. E. A.; NUNES, L. G. Artisanal fisheries of the Xingu river basin in Brazilian Amazon. Brazilian Journal of Biology, v. 75, n. 3 (suplemento), p. s125-137, 2016. Disponível em: <http://dx.doi.org/10.1590/15196984.00314BM>.

JUNK, W. J.; BAYLEY, P. B.; SPARKS, R. E. The flood pulse concept in riverfloodplain systems. In: DODGE, D. P. (Ed.). Proceedings of the International Large River Symposium. Canadian Special Publication of Fisheries and Aquatic Sciences, v. 106, p. 110-127, 1989. Disponível em: $<$ https://www.dfompo.gc.ca/Library/111846.pdf>.

KEPPELER, F. W.; SOUZA, A. C.; HALLWASS, G.; BEGOSSI, A.; ALMEIDA, M. C. et al. Ecological influences of human population size and distance to urban centres on fish communities in tropical lakes. Aquatic Conservation: Marine Freshwater Ecosystem, p. 1-14, 2018. doi: 10.1002/aqc.2910

LOPES, G. C. S.; CATARINO, M. F.; LIMA, A. C.; FREITAS, C. E. C. Small-scale fisheries in the Amazon Basin: general patterns and diversity of fish landings in five sub-basins. Boletim do Instituto de Pesca, São Paulo, v. 42, n. 4, p. 889-900, $2016 . \quad$ Disponível em: <https://www.pesca.sp.gov.br/boletim/index.php/bip/article/view/1186/1165>.

MCCLUSKEY, S. M.; LEWISON, R. B. Quantifying fishing effort: a synthesis of current methods and their applications. Fish and Fisheries, n. 9, p. 188-200, 2008.

MCGRATH, D. D.; CASTRO, F.; FUTEMMA, B. D. A.; CALABRIA, J. Fisheries and the evolution on the lower Amazon Floodplain. Human Ecology, v. 21, n. 2, 1993. Disponível em: <https://doi.org/10.1007/BF00889358>.

MCGRATH, D. G.; SILVA, U. L.; CROSSA, N. M. M. A traditional floodplain fishery of the lower Amazon river, Brazil. Naga, The ICLARM Quaterly, v. 21, n. 1, p. 4-11, 1998. Disponível em: <http://aquaticcommons.org/9338/>.

MCGRATH, D. G.; CASTELLO, L.; ALMEIDA, O. T.; ESTUPINÃN, G. M. B. Market formalization, governance, and the integration of community fisheries in the Brazilian 
Amazon. Society and Natural Resources: An International Journal, v. 28, n. 5, p. 513-529, 2015. doi: 10.1080/08941920.2015.1014607

PEREIRA, D. V.; SILVA, L. F.; SOUSA, K. N. S. Distribuição especial dos sítios de captura registradas nos polos de desembarque pesqueiro no município de Santarém (Pará-Brasil). Biota Amazônia, v. 9, n. 1, p. 43-47, 2019. Disponível em: < http://dx.doi.org/10.18561/2179-5746/biotaamazonia.v9n1p43-47>.

SCHONS, S. Z. Determinantes da participação dos domicílios em acordos de pesca no Baixo Amazonas, estado do Pará. Revista de Ciências da Sociedade, v. 2, n. 4, p. 32-53, 2018. Disponível em:

<http://www.ufopa.edu.br/portaldeperiodicos/index.php/revistacienciasdasociedade/a rticle/view/901>

SILVANO, R. M.; HALLWASS, G.; JURAS, A. A.; LOPES, P. F. M. Assessment of efficiency and impacts of gillnets on fish conservation in a tropical freshwater fishery. Aquatic Conservation: marine and freshwater ecosystems, 2016. doi: 10.1002/aqc.2687

SMITH, N. J. H. A pesca no rio Amazonas. Manaus, 1979. 